УДК 517.968.4

\title{
НЕЛИНЕЙНЫЕ ИНТЕГРАЛЬНЫЕ УРАВНЕНИЯ ТИПА СВЕРТКИ В КОМПЛЕКСНЫХ ПРОСТРАНСТВАХ
}

\author{
C.H. АСХАБОВ
}

\begin{abstract}
Аннотация. Изучаются различные классы нелинейных интегральных уравнений типа свертки, возникающих в теории следящих систем, моделях популяционной генетики и других. Методом монотонных (по Браудеру-Минти) операторов доказаны глобальные теоремы о существовании, единственности и оценках решений рассматриваемых уравнений в комплексных пространствах Лебега $L_{p}(\mathbb{R})$ при достаточно легко обозримых ограничениях на нелинейности. При этом, в зависимости от рассматриваемого класса уравнений, предполагается, что либо $p \in(1,2]$, либо $p \in[2, \infty)$. Условия, накладываемые на нелинейности, являются необходимыми и достаточными для того, чтобы порождаемые ими операторы суперпозиции действовали из пространства $L_{p}(\mathbb{R})$, $1<p<\infty$, в сопряженное с ним пространство $L_{q}(\mathbb{R}), q=p /(p-1)$, и были монотонными. В случае пространства $L_{2}(\mathbb{R})$, комбинированием метода монотонных операторов и принципа сжимающих отображений, показано, что решения могут быть найдены методом последовательных приближений пикаровского типа и приведены оценки скорости их сходимости. Доказательства существенно используют установленные в работе критерий положительности (по Бохнеру) линейного интегрального оператора свертки в комплексном пространстве Лебега $L_{p}(\mathbb{R})$ при $1<p \leqslant 2$ и коэрцитивность оператоpa, обратного к нелинейному оператору Немыцкого. Полученные результаты в рамках пространства $L_{2}(\mathbb{R})$ охватывают, в частности, линейные интегральные уравнения типа свертки.
\end{abstract}

Ключевые слова: нелинейные интегральные уравнения, оператор свертки, критерий положительности, монотонный оператор, коэрцитивный оператор.

Mathematics Subject Classification: 45G10, 47J05

\section{1. ВВЕДЕНИЕ}

Решение многих задач современной математики, физики, механики и биологии приводят к нелинейным интегральным уравнениям типа свертки (см. монографии [1], [2] и приведенную в них библиографию). Например, общий класс нелинейных сервомеханизмов (следящих систем) описывается рассматриваемым в данной работе нелинейным интегральным уравнением типа свертки вида [3]:

$$
u(x)+\int_{-\infty}^{\infty} h(x-t) F[t, u(t)] d t=f(x),
$$

которое возникает также в теории электрических сетей (сигнальной трансмиссии через общую электрическую сеть), содержащих нелинейные элементы (нелинейный резистор) [4].

S.N. Askhabov, Nonlinear convolution type integral equations in complex spaces.

(C) Асхабов C.H. 2021.

Работа поддержана РФФИ (грант 18-41-200001) и публикуется в рамках выполнения государственного задания в соответствии с Дополнительным соглашением от 07.07.2020 № 075-03-2020-239/2 реестр № 248 КБК 01104730290059611 (проект «Нелинейные сингулярные интегро-дифференциальные уравнения и краевые задачи»).

Поступила 29 ноября 2020 2. 
При $f(x)=0$ уравнение вида (1.1) описывает детерменистические модели пространственного распространения эпидемии, а также используется как математическая модель некоторых инфекционных заболеваний или как уравнение роста некоторых видов популяции [5], 6].

Известно, что теория линейных интегральных уравнений типа свертки в настоящее время достаточно хорошо разработана и ее основные результаты приведены, например, в монографии [7]. Что касается теории нелинейных интегральных уравнений типа свертки, то она находится в стадии развития и отличается от соответствующей линейной теории не только по методам исследования, но и по характеру получаемых результатов (подробнее, см. [1], 2]).

В последние десятилетия при исследовании нелинейных уравнений с положительными операторами широко используется метод монотонных операторов. K сожалению, как отмечено в монографии [8, глава 8, п. 8.3] оба термина «положительный» и «монотонный» используются в функциональном анализе в нескольких различных смыслах. Так, в работах М.А. Красносельского, И.А. Бахтина и других (см., например, [9]) развита глобальная теория положительных решений нелинейных уравнений с монотонными (по Красносельскому) операторами в банаховых пространствах с конусами, а в работах Г. Минти, Ф. Браудера, Р.И. Качуровского, М.М. Вайнберга и других (см., например, [10], [11]) построена теория решений (произвольного знака) нелинейных уравнений с монотонными (по Браудеру-Минти) операторами в рефлексивных пространствах. K настоящему времени опубликовано значительно больше работ, посвященных исследованию нелинейных интегральных уравнений типа свертки с монотонными по Красносельскому операторами, чем с монотонными по Браудеру-Минти операторами.

В данной работе методом монотонных (по Браудеру-Минти) операторов доказаны глобальные теоремы о существовании, единственности и оценках решений для трех различных классов нелинейных интегральных уравнений типа свертки в комплексных пространствах Лебега $L_{p}(\mathbb{R})$. При $p=2$ показано, что решения могут быть найдены методом последовательных приближений пикаровского типа и приведены оценки скорости их сходимости. Доказательства существенно используют установленный в работе критерий положительности (по Бохнеру) интегрального оператора свертки. Полученные результаты в рамках пространства $L_{2}(\mathbb{R})$ охватывают, в частности, линейные интегральные уравнения типа свертки.

Следует отметить, что в работе [12] изучены различные классы нелинейных интегральных уравнений типа свертки в вещественных пространствах Лебега $2 \pi$-периодических функций $L_{p}(-\pi, \pi)$ при любых значениях $p \in(1, \infty)$. Исследование этих уравнений в случае комплексных пространств Лебега $L_{p}(\mathbb{R})$ вызывает дополнительные трудности, связанные, в частности, с тем, что, в отличие от пространств $L_{p}(-\pi, \pi)$, они не являются вложенными друг в друга в зависимости от значений $p$, а также нахождением условий положительности оператора свертки и условий монотонности и коэрцитивности оператора суперпозиции. Оказалось, что эти условия существенно отличаются от известных в случае вещественных пространств $L_{p}(-\pi, \pi)$. Эти отличия приводят к тому, что, в зависимости от рассматриваемого класса нелинейных интегральных уравнений типа свертки, в случае комплексных пространств $L_{p}(\mathbb{R})$ приходится предполагать, что либо $p \in(1,2]$, либо $p \in[2, \infty)$.

\section{2. КРИТЕРИЙ ПОЛОЖИТЕЛЬНОСТИ ИНТЕГРАЛЬНОГО ОПЕРАТОРА СВЕРТКИ В КОМПЛЕКСНЫХ ПРОСТРАНСТВАХ ЛЕБЕГА}

Как известно [13, глава 9], теория непрерывных положительно-определенных (по Бохнеру) функций в настоящее время достаточно разработана и может рассматриваться как 
одно из исходных орудий построения гармонического анализа, играя, в частности, важную роль в теории локально-компактных групп. С понятием положительно-определенной функции тесно связано понятие положительного оператора, нашедшее многочисленные применения при исследовании как линейных, так и нелинейных интегральных и дискретных уравнений в банаховых пространствах [1], [14, [15], [16].

В монографии [1, § 10] доказано, что для положительности в вещественном пространстве Лебега $L_{p}(\mathbb{R})$, где $1<p \leqslant 2$, интегрального оператора свертки $H u=h * u$ необходимо и достаточно, чтобы косинус-преобразование Фурье $\widehat{h}_{c}(x)$ его ядра $h \in L_{1}(\mathbb{R}) \cap L_{p /[2(p-1)]}(\mathbb{R})$ было неотрицательной функцией на положительной полуоси $[0, \infty)$.

В данном пункте установлено, что интегральный оператор свертки $H$ является положительным в комплексном пространстве Лебега $L_{p}(\mathbb{R})$ тогда и только тогда, когда реальная часть преобразования Фурье его ядра является неотрицательной функцией на всей числовой оси $\mathbb{R}$.

Итак, рассмотрим в комплексном пространстве Лебега $L_{p}(\mathbb{R}), 1<p<\infty$, интегральный оператор свертки

$$
(H u)(x)=\int_{-\infty}^{\infty} h(x-t) u(t) d t=(h * u)(x)
$$

где ядро $h \in L_{1}(\mathbb{R})$. Для $u \in L_{p}(\mathbb{R})$ и $v \in L_{q}(\mathbb{R}), q=p /(p-1)$, введем обозначения:

$$
\|u\|_{p}=\left(\int_{-\infty}^{\infty}|u(x)|^{p} d x\right)^{1 / p} \quad \text { и }\langle u, v\rangle=\int_{-\infty}^{\infty} u(x) \cdot \overline{v(x)} d x .
$$

Если $p=q=2$, то $\langle u, v\rangle=(u, v)$ есть обычное скалярное произведение в гильбертовом пространстве $L_{2}(\mathbb{R})$.

Обозначим через $\widehat{u}(x)$ преобразование Фурье функции $u \in L_{2}(\mathbb{R})$ (приводимые ниже сведения из теории преобразования Фурье см., например, в [17, глава VIII]):

$$
\widehat{u}(x)=\underset{N \rightarrow \infty}{\lim .} \frac{1}{\sqrt{2 \pi}} \int_{-N}^{N} u(t) e^{-i x t} d t
$$

где символ $\lim _{N \rightarrow \infty}$ означает предел в среднем с показателем $p=2$ (т.е. в среднем квадратичном).

Известно, что $\widehat{u} \in L_{2}(\mathbb{R})$, если $u \in L_{2}(\mathbb{R})$ и для любых $u, v \in L_{2}(\mathbb{R})$ справедливо обобщенное равенство Парсеваля $(u, v)=(\widehat{u}, \widehat{v})$, т.е.

$$
\int_{-\infty}^{\infty} u(x) \cdot \overline{v(x)} d x=\int_{-\infty}^{\infty} \widehat{u}(x) \cdot \overline{\widehat{v}(x)} d x
$$

где черта сверху означает комплексное сопряжение. Кроме того, если ядро $h \in L_{1}(\mathbb{R})$, а $u \in L_{2}(\mathbb{R})$, то для преобразования Фурье свертки справедливо равенство:

$$
\widehat{(h * u)}(x)=\sqrt{2 \pi} \widehat{h}(x) \cdot \widehat{u}(x),
$$

где $\widehat{h}(x)=\frac{1}{\sqrt{2 \pi}} \int_{-\infty}^{\infty} h(t) e^{-i x t} d t$ (поскольку $h(x) \in L_{1}(\mathbb{R})$, то выражение 2.1 упрощается). 
Лемма 2.1. Пусть $1<p \leqslant 2$ и ядро $h \in L_{1}(\mathbb{R}) \cap L_{q / 2}(\mathbb{R})$, где $q=p /(p-1)$. Для того, чтобы оператор свертки $H$ (действующий непрерывно из $L_{p}(\mathbb{R})$ в $L_{q}(\mathbb{R})$ ) был положительным, т.е. выполнялось неравенство:

$$
\operatorname{Re}\langle H u, u\rangle=\operatorname{Re} \int_{-\infty}^{\infty}\left(\int_{-\infty}^{\infty} h(x-t) u(t) d t\right) \overline{u(x)} d x \geqslant 0, \quad \forall u \in L_{p}(\mathbb{R}),
$$

необходимо и достаточно, чтобъ выполнялось условие:

$$
\operatorname{Re} \widehat{h}(x)=\frac{1}{\sqrt{2 \pi}} \operatorname{Re} \int_{-\infty}^{\infty} h(t) e^{-i x t} d t \geqslant 0, \quad \forall x \in \mathbb{R} .
$$

Доказательство. Достаточность. Так как $h \in L_{q / 2}(\mathbb{R})$, то из неравенства Юнга (см., например, [1, теорема 4.4]) непосредственно вытекает оценка

$$
\|H u\|_{q} \leqslant\|h\|_{q / 2}\|u\|_{p}, \quad \forall u \in L_{p}(\mathbb{R}) .
$$

Значит, оператор свертки $H$ действует непрерывно из $L_{p}(\mathbb{R})$ в $L_{q}(\mathbb{R})$.

Докажем положительность оператора свертки $H$. Для этого рассмотрим отдельно два случая: $p=2$ и $1<p<2$.

1). Пусть $p=2$. Тогда $q=2$ и, по условию леммы 2.1, $h \in L_{1}(\mathbb{R})$. Значит, в силу неравенства (2.6), оператор свертки $H$ действует непрерывно из $L_{2}(\mathbb{R})$ в $L_{2}(\mathbb{R})$. Используя равенства 2.2) и 2.3), имеем

$$
\begin{aligned}
(H u, u) & =\int_{-\infty}^{\infty}\left(\int_{-\infty}^{\infty} h(x-t) u(t) d t\right) \overline{u(x)} d x=\int_{-\infty}^{\infty}(h * u)(x) \cdot \overline{u(x)} d x \\
& =\int_{-\infty}^{\infty} \widehat{(h * u)}(x) \cdot \overline{\widehat{u}(x)} d x=\sqrt{2 \pi} \int_{-\infty}^{\infty} \widehat{h}(x) \cdot \widehat{u}(x) \cdot \overline{\widehat{u}(x)} d x=\sqrt{2 \pi} \int_{-\infty}^{\infty} \widehat{h}(x) \cdot|\widehat{u}(x)|^{2} d x
\end{aligned}
$$

Значит,

$$
\operatorname{Re}(H u, u)=\sqrt{2 \pi} \int_{-\infty}^{\infty} \operatorname{Re} \widehat{h}(x) \cdot|\widehat{u}(x)|^{2} d x
$$

Из равенства 2.7) следует, что $\operatorname{Re}(H u, u) \geqslant 0$ для любого $u \in L_{2}(\mathbb{R})$, если $\operatorname{Re} \widehat{h}(x) \geqslant 0$ для почти всех $x \in \mathbb{R}$. Так как, по теореме Римана-Лебега, $\widehat{h}(x)$ есть непрерывная на всей оси $\mathbb{R}$ функция, то условие, что $\operatorname{Re} \widehat{h}(x) \geqslant 0$ для почти всех $x \in \mathbb{R}$ равносильно условию, что $\operatorname{Re} \widehat{h}(x) \geqslant 0$ для всех $x \in \mathbb{R}$.

Таким образом, если $h \in L_{1}(\mathbb{R})$ и $\operatorname{Re} \widehat{h}(x) \geqslant 0$ для всех $x \in \mathbb{R}$, то

$$
\operatorname{Re}(H u, u) \geqslant 0, \quad \forall u \in L_{2}(\mathbb{R}) .
$$

2). Пусть теперь $1<p<2, h(x) \in L_{1}(\mathbb{R}) \cap L_{q / 2}(\mathbb{R})$ и выполняется условие (2.5). Так как $h(x) \in L_{1}(\mathbb{R})$, то на основании неравенства 2.8$)$, имеем

$$
\operatorname{Re}\langle H u, u\rangle \geqslant 0, \quad \forall u(x) \in L_{2}(\mathbb{R}) \cap L_{p}(\mathbb{R}) .
$$

C другой стороны, в силу неравенства Гельдера и неравенства Юнга (2.6), для любого $u \in L_{p}(\mathbb{R})$ имеем:

$$
|\langle H u, u\rangle| \leqslant\|H u\|_{q}\|u\|_{p} \leqslant\|h\|_{q / 2}\|u\|_{p}^{2},
$$

т.е. функционал $\langle H u, u\rangle$ непрерывен в $L_{p}(\mathbb{R})$. Поскольку множество $L_{2}(\mathbb{R}) \cap L_{p}(\mathbb{R})$ всюду плотно в классе $L_{p}(\mathbb{R})$ и $\langle H u, u\rangle$ есть непрерывный функционал, то неравенство $\operatorname{Re}\langle H u, u\rangle \geqslant 0$, т.е. неравенство (2.9), выполняется для любого $u \in L_{p}(\mathbb{R})$. 
Необходимость. Докажем теперь, что условие (2.5) так же и необходимо для положительности оператора $H$. Пусть неравенство 2.4 выполнено. Нужно доказать, что тогда $\operatorname{Re} \widehat{h}(x) \geqslant 0$ для всех $x \in \mathbb{R}$, т.е. выполнено условие 2.5$)$. Допустим противное, что условие (2.5) не выполняется, т.е. существует точка $x_{0} \in \mathbb{R}$ такая, что $\operatorname{Re} \widehat{h}\left(x_{0}\right)<0$. Поскольку, по теореме Римана-Лебега, $\operatorname{Re} \widehat{h}(x)$ есть непрерывная на всей оси $\mathbb{R}$ функция, то найдется достаточно малая $\varepsilon$-окрестность $U_{\varepsilon}\left(x_{0}\right)=\left\{x:\left|x-x_{0}\right|<\varepsilon\right\}, \varepsilon>0$, точки $x_{0}$ такая, что будет выполняться неравенство

$$
\operatorname{Re} \widehat{h}(x)<0, \quad \forall x \in U_{\varepsilon}\left(x_{0}\right) .
$$

Выберем целую функцию $u$ такую, что $u_{\mid \mathbb{R}} \in L_{p}(\mathbb{R}), \widehat{u}(x)=0$ для $x \notin U_{\varepsilon}\left(x_{0}\right)$ и $\widehat{u}(x) \neq 0$, если $x \in U_{\varepsilon}\left(x_{0}\right)$. Тогда, учитывая, что $\forall x \in U_{\varepsilon}\left(x_{0}\right)$ выполняются строгие неравенства $\operatorname{Re} \widehat{h}(x)<0$ и $|\widehat{u}(x)|>0$, по формуле 2.7) для так выбранной функции $u(x)$ получим

$$
\operatorname{Re}\langle H u, u\rangle=\sqrt{2 \pi} \int_{-\infty}^{\infty} \operatorname{Re} \widehat{h}(x) \cdot|\widehat{u}(x)|^{2} d x=\sqrt{2 \pi} \int_{U_{\varepsilon}\left(x_{0}\right)} \operatorname{Re} \widehat{h}(x) \cdot|\widehat{u}(x)|^{2} d x<0,
$$

что противоречит неравенству (2.4), которое по предположению выполнено для любого $u \in L_{p}(\mathbb{R})$.

При исследовании нелинейных интегральных уравнений типа свертки вида (1.1) нам понадобится также следующая лемма, двойственная лемме 2.1 .

Лемма 2.2. Пусть $p \geqslant 2$ и ядро $h \in L_{1}(\mathbb{R}) \cap L_{p / 2}(\mathbb{R})$. Для того, итобъ оператор свертки $H$ (действующий непрерывно из $L_{q}(\mathbb{R})$ в $\left.L_{p}(\mathbb{R})\right)$ был положительным, необходимо и достаточно, чтобы выполнялось условие (2.5).

Доказательство леммы 2.2 проводится точно так же, как и доказательство леммы 2.1 .

\section{3. ТЕОРЕМЫ СУЩЕСТВОВАНИЯ И ЕДИНСТВЕННОСТИ РЕШЕНИЯ В $L_{p}(\mathbb{R})$}

Приведем определения, обозначения и некоторые результаты из теории монотонных (по Браудеру-Минти) операторов, используемые в данной статье.

Пусть $X$ - комплексное банахово пространство и $X^{*}$ - сопряженное с ним пространство. Обозначим через $\langle y, x\rangle$ значение линейного непрерывного функционала $y \in X^{*}$ на элементе $x \in X$, а через $\|\cdot\|$ и $\|\cdot\|_{*}$ нормы в $X$ и $X^{*}$, соответственно. В частности, если $X$ есть гильбертово пространство $H$, то $\langle y, x\rangle$ совпадает со скалярным произведением $(y, x)$, где $x, y \in H$.

Определение 3.1. Пусть $u, v \in X$ - произвольные элементы. Оператор $A: X \rightarrow X^{*}$ (т.е. действующий из $X$ в $\left.X^{*}\right)$ называетсл:

монотонным, если $\operatorname{Re}\langle A u-A v, u-v\rangle \geqslant 0 ;$

строго монотонным, если $\operatorname{Re}\langle A u-A v, u-v\rangle>0$ nрu $u \neq v$;

сильно монотонным, если $\operatorname{Re}\langle A u-A v, u-v\rangle \geqslant m \cdot\|u-v\|^{2}, m>0$;

коэрцитивным, если

$$
\lim _{\|u\| \rightarrow \infty} \frac{\operatorname{Re}\langle A u, u\rangle}{\|u\|}=\infty
$$

липшиц-непрерывным, если $\|A u-A v\|_{*} \leqslant M \cdot\|u-v\|, M>0$;

хеминепрерывным, если функиия $s \rightarrow\langle A(u+s \cdot v), w\rangle$ непрерывна на $[0,1]$ при любых фиксированньх $u, v, w \in X$;

деминепрерывным, если из сильной сходимости $u_{n} \rightarrow u$ в $X$ следует слабая сходимость $A u_{n} \rightarrow A u$ в $X^{*}$. 
Если $A$ - линейный оператор, то определение монотонного, строго монотонного и сильно монотонного оператора совпадает, соответственно, с определением положительного, строго положительного и сильно положительного (положительно определенного) оператора [10, $\S 1]$.

Известно [11, Замечание 1.8, глава III], что для монотонных операторов понятия хеминепрерывность и деминепрерывность, являющиеся ослаблением обычного понятия непрерывности, совпадают.

Основной в теории монотонных операторов является следующая теорема Ф. Браудера и Г. Минти [11, §2, глава III] (в случае комплексных пространств $X$ она доказана в [10, § 18] и [18, Теорема 1.1, глава II]).

Теорема 3.1. Пусть $X$ есть рефлексивное банахово пространство и оператор $A$ : $X \rightarrow X^{*}$ является хеминепрерывным, монотонным и коэриитивным. Тогда уравнение $A u=f$ имеет решение $u^{*} \in X$ для любого $f \in X^{*}$. Это решение единственно в $X$, если A - строго монотонный оператор.

Обозначим через $\mathbb{C}$ множество всех комплексных чисел, а через $L_{p}^{+}(\mathbb{R})$ - множество всех неотрицательных функций из $L_{p}(\mathbb{R})$. Введем в рассмотрение нелинейный оператор суперпозиции (часто называемый оператором Немыцкого [10]) $(F u)(x)=F[x, u(x)]$, порожденный комплекснозначной функцией $F(x, z): \mathbb{R} \times \mathbb{C} \rightarrow \mathbb{C}$, удовлетворяющей известным условиям Каратеодори: она измерима по $x \in \mathbb{R}$ при каждом фиксированном $z \in \mathbb{C}$ и непрерывна по $z$ почти для всех $x \in \mathbb{R}$.

Выпишем для удобства ссылок все ограничения на функцию $F(x, z)$, определяющую нелинейность исследуемых в этом пункте уравнений. Именно в зависимости от рассматриваемого класса нелинейных интегральных уравнений типа свертки, будем накладывать на нелинейность $F(x, z)$ либо условия 3.1$)-3.3)$, либо условия 3.4$)-3.6)$, где всюду $p \in(1, \infty)$ :

3.1) существуют $c \in L_{q}^{+}(\mathbb{R})$ и $d_{1}>0$ такие, ито для почти всех $x \in \mathbb{R}$ и любого z $\in \mathbb{C}$ выполняется неравенство:

$$
|F(x, z)| \leqslant c(x)+d_{1} \cdot|z|^{p-1}
$$

3.2) для почти всех $x \in \mathbb{R}$ и всех $z_{1}, z_{2} \in \mathbb{C}$ выполняется неравенство:

$$
\operatorname{Re}\left\{\left[F\left(x, z_{1}\right)-F\left(x, z_{2}\right)\right] \cdot \overline{\left(z_{1}-z_{2}\right)}\right\} \geqslant 0
$$

3.3) существуют $D \in L_{1}^{+}(\mathbb{R})$ и $d_{2}>0$ такие, что для почти всех $x \in \mathbb{R}$ и всех $z \in \mathbb{C}$ выполняется неравенство:

$$
\operatorname{Re}\{F(x, z) \cdot \bar{z}\} \geqslant d_{2} \cdot|z|^{p}-D(x)
$$

3.4) существуют $g \in L_{p}^{+}(\mathbb{R})$ и $d_{3}>0$ такие, что для почти всех $x \in \mathbb{R}$ и любого z $\in \mathbb{C}$ выполняется неравенство:

$$
|F(x, z)| \leqslant g(x)+d_{3} \cdot|z|^{1 /(p-1)} ;
$$

3.5) для почти всех $x \in \mathbb{R}$ и всех $z_{1}, z_{2} \in \mathbb{C}$ выполняется неравенство:

$$
\operatorname{Re}\left\{\left[F\left(x, z_{1}\right)-F\left(x, z_{2}\right)\right] \cdot \overline{\left(z_{1}-z_{2}\right)}\right\}>0
$$

3.6) существуют $D \in L_{1}^{+}(\mathbb{R})$ и $d_{4}>0$ такие, что для почти всех $x \in \mathbb{R}$ и всех $z \in \mathbb{C}$ выполняется неравенство:

$$
\operatorname{Re}\{F(x, z) \cdot \bar{z}\} \geqslant d_{4} \cdot|z|^{p /(p-1)}-D(x) .
$$

Заметим, что если выполнены условия 3.1)-3.3), то оператор Немыцкого $F$, порожденный функцией $F(x, z)$, действует из $L_{p}(\mathbb{R})$ в $L_{q}(\mathbb{R})$ и является непрерывным, монотонным и коэрцитивным оператором. Если же выполнены условия 3.4)-3.6), то оператор $F$ действует 
наоборот из $L_{q}(\mathbb{R})$ в $L_{p}(\mathbb{R})$ и является непрерывным, строго монотонным и коэрцитивным оператором (см., например, [1, § 2]).

Простейшим примером функции $F(x, z)$, удовлетворяющей условиям 3.1)-3.3), может служить $F(x, z)=z \cdot|z|^{p-2}$, где $p \geqslant 2$ есть любое число. В самом деле, выполнение условий $3.1)$ и 3.3) для такой функции очевидно, при этом $\|F u\|_{q}=\|u\|_{p}^{p-1}, \operatorname{Re}\langle F u, u\rangle=\|u\|_{p}^{p}$. Проверим выполнимость условия 3.2) при $p>2$ (выполнение этого условия при $p=2$ очевидно). Для любых $z_{1}=x_{1}+i y_{1}$ и $z_{2}=x_{2}+i y_{2}$, имеем:

$$
\left[F\left(x, z_{1}\right)-F\left(x, z_{2}\right)\right] \cdot \overline{\left[z_{1}-z_{2}\right]}=\left|z_{1}\right|^{p}-\left|z_{1}\right|^{p-2} z_{1} \overline{z_{2}}-\left|z_{2}\right|^{p-2} z_{2} \overline{z_{1}}+\left|z_{2}\right|^{p} .
$$

Так как

$$
\operatorname{Re}\left(z_{1} \overline{z_{2}}\right)=\operatorname{Re}\left(z_{2} \overline{z_{1}}\right)=x_{1} x_{2}+y_{1} y_{2} \leqslant \frac{1}{2}\left(x_{1}^{2}+x_{2}^{2}+y_{1}^{2}+y_{2}^{2}\right)=\frac{1}{2}\left(\left|z_{1}\right|^{2}+\left|z_{2}\right|^{2}\right),
$$

TO

$$
\begin{aligned}
\operatorname{Re}\left\{\left[F\left(x, z_{1}\right)-F\left(x, z_{2}\right)\right] \cdot \overline{\left[z_{1}-z_{2}\right]}\right\} & =\left|z_{1}\right|^{p}+\left|z_{2}\right|^{p}-\left(x_{1} x_{2}+y_{1} y_{2}\right)\left(\left|z_{1}\right|^{p-2}+\left|z_{2}\right|^{p-2}\right) \\
& \geqslant\left|z_{1}\right|^{p}+\left|z_{2}\right|^{p}-\frac{1}{2}\left(\left|z_{1}\right|^{2}+\left|z_{2}\right|^{2}\right)\left(\left|z_{1}\right|^{p-2}+\left|z_{2}\right|^{p-2}\right) \\
& =\frac{1}{2}\left(\left|z_{1}\right|^{p-2}-\left|z_{2}\right|^{p-2}\right)\left(\left|z_{1}\right|^{2}-\left|z_{2}\right|^{2}\right) \geqslant 0
\end{aligned}
$$

т.е. выполнено условие 3.2).

Рассмотрим сначала наиболее простое для исследования методом монотонных операторов уравнение, в которое нелинейный оператор суперпозиции и линейный оператор свертки входят как слагаемые.

Теорема 3.2. Пусть $1<p \leqslant 2$, ядро $h \in L_{1}(\mathbb{R}) \cap L_{q / 2}(\mathbb{R})$ и удовлетворлет условию 2.5). Если нелинейность $F(x, z)$ удовлетворяет условиям 3.1)-3.3), то уравнение

$$
F[x, u(x)]+\int_{-\infty}^{\infty} h(x-t) u(t) d t=f(x)
$$

имеет решение $u^{*} \in L_{p}(\mathbb{R})$ при любом $f \in L_{q}(\mathbb{R})$. Это решение единственно, если вместо условия 3.2) выполнено условие 3.5). При этом, если условие 3.3) выполнено с $D(x)=0$, справедлива оценка:

$$
\left\|u^{*}\right\|_{p} \leqslant\left(d_{2}^{-1} \cdot\|f\|_{q}\right)^{1 /(p-1)} .
$$

Доказательство. Запишем уравнение (3.1) в операторном виде: $A u=f$, где $A=F+H$. Из условий 3.1)-3.3) вытекает, что оператор суперпозиции $F$, порожденный функцией $F(x, z)$, действует из $L_{p}(\mathbb{R})$ в $L_{q}(\mathbb{R})$ и является непрерывным, монотонным и коэрцитивным оператором, причем он является строго монотонным оператором, если выполнено условие 3.5). Из леммы 2.1 вытекает, что оператор свертки $H$ также действует из $L_{p}(\mathbb{R})$ в $L_{q}(\mathbb{R})$ и является непрерывным и положительным (или, что то же самое, монотонным, в силу его линейности) оператором. Значит, оператор $A$ действует непрерывно (а значит и хеминепрерывно) из рефлексивного пространства $L_{p}(\mathbb{R})$ в сопряженное с ним пространство $L_{q}(\mathbb{R})$ и является монотонным и коэрцитивным оператором, причем он является строго монотонным оператором, если выполнено условие 3.5). Поэтому, в силу теоремы 3.1 (БраудераМинти), уравнение $A u=f$, а значит и уравнение $(3.1)$, имеет решение $u^{*} \in L_{p}(\mathbb{R})$ и это решение единственно, если выполнено условие 3.5). 
Осталось доказать оценку нормы решения. Пусть $u^{*} \in L_{p}(\mathbb{R})$ есть решение уравнения (3.1), т.е. $A u^{*}=f$. Используя сначала условие 3.3$)$ при $D(x)=0$, а затем положительность оператора свертки $H$, равенство $A u^{*}=f$ и неравенство Гельдера, имеем

$$
\begin{array}{r}
d_{2} \cdot\left\|u^{*}\right\|_{p}^{p} \leqslant \operatorname{Re}\left\langle F u^{*}, u^{*}\right\rangle \leqslant \operatorname{Re}\left\langle F u^{*}, u^{*}\right\rangle+\operatorname{Re}\left\langle H u^{*}, u^{*}\right\rangle= \\
=\operatorname{Re}\left\langle A u^{*}, u^{*}\right\rangle=\operatorname{Re}\left\langle f, u^{*}\right\rangle \leqslant\|f\|_{q} \cdot\left\|u^{*}\right\|_{p}
\end{array}
$$

откуда непосредственно вытекает доказываемая оценка.

Следует отметить, что оператор Немыцкого $F$ является одним из немногих нелинейных операторов, для которых известны критерии их поведения. Так, например, условие 3.1) необходимо и достаточно для того, чтобы оператор $F$ действовал из $L_{p}(\mathbb{R})$ в сопряженное с ним пространство $L_{q}(\mathbb{R}), q=p /(p-1)$, и был непрерывным, а условие 3.2$)$ необходимо и достаточно для того, чтобы этот оператор был монотонным (ср. [10]). Благодаря этим критериям, при выполнении условий 3.1), 3.2) и 3.5), удается доказать существование, хеминепрерывность, строгую монотонность и, что особенно важно для доказательства следующих двух теорем, корцитивность обратного оператора $F^{-1}$.

Приступим теперь к исследованию уравнения вида $(1.1)$, относящемуся к известному классу нелинейных интегральных уравнений типа Гаммерштейна. Следует отметить, что метод монотонных (по Браудеру-Минти) операторов применялся к нелинейным интегральным уравнениям вида (1.1) с общим ядром $k(x, t)$, вместо разностного ядра $h(x-t)$, во многих работах (см., например, [10], [19], [20]), однако в этих работах заведомо предполагается, что линейный интегральный оператор с ядром $k(x, t)$ действует из пространства Лебега в сопряженное с ним пространство и является положительным, но не приводятся условия, при которых этот оператор обладает такими свойствами. В случае разностного ядра $h(x-t)$ указанные условия представлены в следующей теореме.

Теорема 3.3. Пусть $p \geqslant 2$, ядро $h \in L_{1}(\mathbb{R}) \cap L_{p / 2}(\mathbb{R})$ и удовлетворяет условию (2.5). Если нелинейность $F(x, z)$ удовлетворяет условиям 3.1), 3.3) и 3.5), то уравнение

$$
u(x)+\int_{-\infty}^{\infty} h(x-t) F[t, u(t)] d t=f(x)
$$

имеет единственное решение $u^{*} \in L_{p}(\mathbb{R})$ при любом $f \in L_{p}(\mathbb{R})$. При этом, если условия 3.1) и 3.3) выполняются с $c(x)=0$ u $D(x)=0$, справедлива оценка:

$$
\left\|u^{*}\right\|_{p} \leqslant \frac{d_{1}}{d_{2}} \cdot\|f\|_{p}
$$

Доказательство. Из условий 3.1), 3.3) и 3.5) вытекает, что оператор суперпозиции $F$ отображает пространство $L_{p}(\mathbb{R})$ на все пространство $L_{q}(\mathbb{R})$, непрерывен, строго монотонен и коэрцитивен. В силу леммы 2.1 из [1], существует обратный оператор $F^{-1}$, отображающий $L_{q}(\mathbb{R})$ на $L_{p}(\mathbb{R})$, хеминепрерывный, строго монотонный и коэрцитивный. С учетом леммы 2.2 имеем, что оператор $A=F^{-1}+H$ действует из $L_{q}(\mathbb{R})$ в $L_{p}(\mathbb{R})$, хеминепрерывен, строго монотонен и коэрцитивен. Значит, на основании теоремы 3.1 (Браудера-Минити), уравнение $A v=f$ имеет единственное решение $v^{*} \in L_{q}(\mathbb{R})$ при любом $f \in L_{p}(\mathbb{R})$. Но тогда $u^{*}=F^{-1} v^{*} \in L_{p}(\mathbb{R})$ является решением уравнения $u+H F u=f$, т.е. данного уравнения (3.2) и оно единственно, в силу условия 3.5$)$.

Осталось доказать оценку нормы решения. Пусть $u^{*} \in L_{p}(\mathbb{R})$ есть решение уравнения $(3.2)$, т.е. $u^{*}+H F u^{*}=f$. Используя сначала условие 3.3$)$ при $D(x)=0$, а затем положительность оператора свертки $H$, равенство $u^{*}+H F u^{*}=f$, неравенство Гельдера и условие 
3.1) при $c(x)=0$, имеем

$$
\begin{aligned}
d_{2} \cdot\left\|u^{*}\right\|_{p}^{p} \leqslant \operatorname{Re}\left\langle u^{*}, F u^{*}\right\rangle & \leqslant \operatorname{Re}\left\langle u^{*}, F u^{*}\right\rangle+\operatorname{Re}\left\langle H F u^{*}, F u^{*}\right\rangle=\operatorname{Re}\left\langle u^{*}+H F u^{*}, F u^{*}\right\rangle \\
& =\operatorname{Re}\left\langle f, F u^{*}\right\rangle \leqslant d_{1} \cdot\|f\|_{p} \cdot\left\|F u^{*}\right\|_{q} \leqslant d_{1} \cdot\|f\|_{p} \cdot\left\|u^{*}\right\|_{p}^{p-1},
\end{aligned}
$$

откуда непосредственно вытекает доказываемая оценка.

Следующая теорема отличается от теорем 3.2 и 3.3 как по характеру ограничений накладываемых на нелинейность $F(x, z)$, так и по структуре доказательства.

Теорема 3.4. Пусть $1<p \leqslant 2$, ядро $h \in L_{1}(\mathbb{R}) \cap L_{q / 2}(\mathbb{R})$ и удовлетворяет условию (2.5). Если нелинейность $F(x, z)$ удовлетворяет условиям 3.4)-3.6), то уравнение

$$
u(x)+F\left[x, \int_{-\infty}^{\infty} h(x-t) u(t) d t\right]=f(x)
$$

имеет единственное решение $u^{*} \in L_{p}(\mathbb{R})$ при любом $f \in L_{p}(\mathbb{R})$. При этом, если условия 3.4) и 3.6) выполняются с $g(x)=0$ и $D(x)=0$, справедлива оценка:

$$
\left\|u^{*}\right\|_{p} \leqslant\left(\frac{d_{3}}{d_{4}}+1\right) \cdot\|f\|_{p}
$$

Доказательство. Из условий 3.4)-3.6) вытекает, что оператор суперпозиции $F$ отображает сопряженное пространство $L_{q}(\mathbb{R})$ на исходное пространство $L_{p}(\mathbb{R})$, в котором ищется решение уравнения (3.3), и является непрерывным, строго монотонным и коэрцитивным оператором. В силу леммы 2.1 из [1], существует обратный оператор $F^{-1}$, отображающий $L_{p}(\mathbb{R})$ на $L_{q}(\mathbb{R})$, хеминепрерывный, строго монотонный и коэрцитивный. С учетом доказанной выше леммы 2.1 имеем, что оператор $A=F^{-1}+H$ действует из $L_{p}(\mathbb{R})$ в $L_{q}(\mathbb{R})$, хеминепрерывен, строго монотонен и коэрцитивен. Значит, на основании теоремы 3.1 (Браудера-Минити), уравнение $A u=H f$, где $H f \in L_{q}(\mathbb{R})$, согласно лемме 2.1. имеет единственное решение $v^{*} \in L_{p}(\mathbb{R})$ при любом $f \in L_{p}(\mathbb{R})$. Но тогда $u^{*}=f-v^{*}$ является решением уравнения $u+F H u=f$, т.е. данного уравнения (3.3), и это решение единственно, в силу условия 3.5).

Осталось доказать оценку нормы решения. Пусть $u^{*} \in L_{p}(\mathbb{R})$ есть решение уравнения $(3.3)$, т.е. $u^{*}+F H u^{*}=f$. Используя условие 3.4$)$ при $g(x)=0$, имеем

$$
\left\|u^{*}-f\right\|_{p}=\left\|F H u^{*}\right\|_{p} \leqslant d_{3} \cdot\left\|H u^{*}\right\|_{q}^{q-1} .
$$

Далее, так как $\left\langle u^{*}+F H u^{*}, H u^{*}\right\rangle=\left\langle f, H u^{*}\right\rangle$, то в силу положительности оператора свертки $H$ получаем

$$
\operatorname{Re}\left\langle F H u^{*}, H u^{*}\right\rangle \leqslant \operatorname{Re}\left\langle u^{*}+F H u^{*}, H u^{*}\right\rangle=\operatorname{Re}\left\langle f, H u^{*}\right\rangle \leqslant\|f\|_{p} \cdot\left\|H u^{*}\right\|_{q} .
$$

С другой стороны, используя условие 3.6$)$ при $D(x)=0$, имеем

$$
\operatorname{Re}\left\langle F H u^{*}, H u^{*}\right\rangle \geqslant d_{4} \cdot\left\|H u^{*}\right\|_{q}^{q} .
$$

Сравнивая неравенства 3.5 и 3.6 , получаем оценку $\left\|H u^{*}\right\|_{q}^{q-1} \leqslant d_{4}^{-1} \cdot\|f\|_{p}$. Но тогда из неравенства (3.4) следует, что $\left\|u^{*}-f\right\|_{p} \leqslant d_{3} \cdot d_{4}^{-1} \cdot\|f\|_{p}$. Поскольку $\left\|u^{*}\right\|_{p}-\|f\|_{p} \leqslant\left\|u^{*}-f\right\|_{p}$, то из последнего неравенства непосредственно вытекает доказываемая оценка.

Заметим, что при $p=2$ теоремы 3.23 .4 охватывают, в частности, и случай линейных интегральных уравнений типа свертки. Кроме того, из полученных в теоремах $3.2[3.4$ оценок для норм решений непосредственно вытекает, что при $f(x) \equiv 0$ уравнения (3.1)(3.3) имеют в $L_{p}(\mathbb{R})$ лишь тривиальное решение $u^{*}(x)=0$. 


\section{4. ПРИБЛИЖЕННОЕ РЕШЕНИЕ УРАВНЕНИЙ В $L_{2}(\mathbb{R})$}

В предыдущем пункте доказаны теоремы 3.23 .4 о существовании, единственности и оценках решений уравнений (3.1)-(3.3). Однако эти теоремы не содержат информации о том, как можно найти решения указанных уравнений. В данном пункте, комбинируя метод монотонных (по Браудеру-Минти) операторов и принцип сжимающих отображений (ср. [11, глава III, теорема 3.4]), доказывается, что решения нелинейных интегральных уравнений типа свертки (3.1)-(3.3) могут быть найдены методом последовательных приближений пикаровского типа в комплексных пространствах $L_{2}(\mathbb{R})$.

Теорема 4.1. Пусть ядро $h \in L_{1}(\mathbb{R})$ и удовлетворяет условию (2.5). Если нелинейность $F(x, z)$ удовлетворяет условиям:

4.1) существует число $M>0$ такое, что для почти всех $x \in \mathbb{R}$ и всех $z_{1}, z_{2} \in \mathbb{C}$ выполняется неравенство:

$$
\left|F\left(x, z_{1}\right)-F\left(x, z_{2}\right)\right| \leqslant M \cdot\left|z_{1}-z_{2}\right| ;
$$

4.2) существует число $m>0$ такое, что для почти всех $x \in \mathbb{R}$ и всех $z_{1}, z_{2} \in \mathbb{C}$ выполняется неравенство:

$$
\operatorname{Re}\left\{\left[F\left(x, z_{1}\right)-F\left(x, z_{2}\right)\right] \cdot \overline{\left(z_{1}-z_{2}\right)}\right\} \geqslant m \cdot\left|z_{1}-z_{2}\right|^{2},
$$

то уравнение (3.1) имеет единственное решение $u^{*} \in L_{2}(\mathbb{R})$ при любом $f \in L_{2}(\mathbb{R})$. Это решение можно найти методом последовательных приближений по формуле:

$$
u_{n}=u_{n-1}-\mu_{1} \cdot\left(F u_{n-1}+H u_{n-1}-f\right), \quad n \in \mathbb{N},
$$

причем справедлива оценка скорости их сходимости:

$$
\left\|u_{n}-u^{*}\right\|_{2} \leqslant \mu_{1} \cdot \frac{\alpha_{1}^{n}}{1-\alpha_{1}} \cdot\left\|F u_{0}+H u_{0}-f\right\|_{2},
$$

где $\mu_{1}=m \cdot\left(M+\|h\|_{1}\right)^{-2}, \alpha_{1}=\sqrt{1-m \cdot \mu_{1}}, u_{0} \in L_{2}(\mathbb{R})$ - прочзволъная функция.

Доказательство. Запишем уравнение (3.1) в операторном виде: $A u=f$, где $A=F+H$. Из условий 4.1) - 4.2) вытекает, что оператор суперпозиции $F$, порожденный функцией $F(x, z)$, действует из $L_{2}(\mathbb{R})$ в $L_{2}(\mathbb{R})$ и является липшиц-непрерывным и сильно монотонным оператором, причем $\forall u, v \in L_{2}(\mathbb{R})$ выполняются неравенства:

$$
\|A u-A v\|_{2} \leqslant\left(M+\|h\|_{1}\right) \cdot\|u-v\|_{2}, \quad \operatorname{Re}(A u-A v, u-v) \geqslant m \cdot\|u-v\|_{2}^{2} .
$$

Поскольку сильная монотонность оператора влечет за собой его строгую монотонность и коэрцитивность, то по теореме 3.1 (Браудера-Минти) уравнение $A u=f$, т.е. данное уравнение (3.1), имеет единственное решение $u^{*} \in L_{2}(\mathbb{R})$.

Осталось доказать, что это решение можно найти методом последовательных приближений по формуле (4.1) с оценкой скорости их сходимости (4.2). Для этого заменим уравнение $A u=f$ на эквивалентное уравнение $u=\Phi u$, где $\Phi u=u-\mu \cdot(A u-f)$ и $\mu>0$ любое (пока) число. Очевидно, что оператор $\Phi$ действует из $L_{2}(\mathbb{R})$ в $L_{2}(\mathbb{R})$ и

$$
\begin{aligned}
\|\Phi u-\Phi v\|_{2}^{2} & =(u-v-\mu \cdot(A u-A v), u-v-\mu \cdot(A u-A v)) \\
& =\|u-v\|_{2}^{2}-2 \mu \cdot \operatorname{Re}(A u-A v, u-v)+\mu^{2} \cdot\|A u-A v\|_{2}^{2} \\
& \leqslant\left(1-2 \mu \cdot m+\mu^{2} \cdot\left(M+\|h\|_{1}\right)^{2}\right) \cdot\|u-v\|_{2}^{2} .
\end{aligned}
$$

Легко проверить, что выражение $1-2 \mu \cdot m+\mu^{2} \cdot\left(M+\|h\|_{1}\right)^{2}$ принимает наименьшее значение, равное $1-m^{2} \cdot\left(M+\|h\|_{1}\right)^{-2}$, при $\mu=\mu_{1}$. Выбрав указанное $\mu$, получаем

$$
\|\Phi u-\Phi v\|_{2} \leqslant \alpha_{1} \cdot\|u-v\|_{2} \text {, }
$$

где $\alpha_{1}=\sqrt{1-m \cdot \mu_{1}} \in(0,1)$. 
Следовательно, оператор $\Phi$ является сжимающим и поэтому формула (4.1) и оценка 4.2 непосредственно вытекают из принципа сжимающих отображений Банаха.

Доказательство теорем, подобных теореме 4.1, для уравнений $(3.2)$ и (3.3) вызывают дополнительные трудности, которые приводят к тому, что последовательные приближения и оценки скорости их сходимости содержат оператор $F^{-1}$, обратный оператору $F$. А именно, справедливы следующие две теоремы.

Теорема 4.2. Пусть ядро $h \in L_{1}(\mathbb{R})$ и удовлетворяет условию (2.5). Если нелинейность $F(x, z)$ удовлетворяет условиям 4.1) и 4.2), то уравнение (3.2) имеет единственное решение $u^{*} \in L_{2}(\mathbb{R})$ при любом $f \in L_{2}(\mathbb{R})$. Это решение можно найти методом последовательных приближений по формуле:

$$
u_{n}=F^{-1} v_{n}, v_{n}=v_{n-1}-\mu_{2} \cdot\left(F^{-1} v_{n-1}+H v_{n-1}-f\right), \quad n \in \mathbb{N},
$$

где $\mu_{2}=m /\left[M \cdot\left(m^{-1}+\|h\|_{1}\right)\right]^{2}, F^{-1}$ есть оператор, обратный $\kappa F$. При этом справедлива оценка скорости сходимости последовательных приближений:

$$
\left\|u_{n}-u^{*}\right\|_{2} \leqslant \frac{\mu_{2}}{m} \cdot \frac{\alpha_{2}^{n}}{1-\alpha_{2}} \cdot\left\|F^{-1} v_{0}+H v_{0}-f\right\|_{2}
$$

где $\alpha_{2}=\sqrt{1-m \cdot \mu_{2} / M^{2}}, v_{0}(x) \in L_{2}(\mathbb{R})$ - произвольная функция.

Доказательство. Из условий 4.1) и 4.2) вытекает, что оператор суперпозиции $F$ действует из $L_{2}(\mathbb{R})$ в $L_{2}(\mathbb{R})$ и является строго монотонным, хеминепрерывным, коэрцитивным и ограниченным оператором, т.е. удовлетворяет всем требованиям теоремы 1.9 [1]. Значит, существует обратный оператор $F^{-1}$, действующий из $L_{2}(\mathbb{R})$ в $L_{2}(\mathbb{R})$, причем (cp. [11, глава III, следствие 2.3]) $\forall u, v \in L_{2}(\mathbb{R})$ выполняются неравенства:

$$
\begin{gathered}
\left\|F^{-1} u-F^{-1} v\right\|_{2} \leqslant \frac{1}{m} \cdot\|u-v\|_{2}, \\
\operatorname{Re}\left(F^{-1} u-F^{-1} v, u-v\right) \geqslant \frac{m}{M^{2}} \cdot\|u-v\|_{2}^{2} .
\end{gathered}
$$

Запишем данное уравнение 3.2 в операторном виде:

$$
u+H F u=f .
$$

В силу теоремы 3.3 оно имеет единственное решение $u^{*} \in L_{2}(\mathbb{R})$. Осталось доказать, что последовательность (4.3) сходится к $u^{*}$ и справедлива оценка (4.4). Для этого наряду с уравнением (4.7) рассмотрим вспомогательное уравнение

$$
\Phi v=f, \quad \text { и } \quad \Phi=F^{-1}+H .
$$

Очевидно, что если $v \in L_{2}(\mathbb{R})$ является решением уравнения 4.8 , то $u^{*}=F^{-1} v^{*} \in L_{2}(\mathbb{R})$ является решением уравнения (4.7). Поэтому достаточно доказать, что уравнение (4.8) имеет единственное решение $v^{*} \in L_{2}(\mathbb{R})$, причем его можно найти по формуле 4.3) и справедлива оценка (4.4). Используя неравенство $\|H u\|_{2} \leqslant\|h\|_{1} \cdot\|u\|_{2}$, являющееся следствием неравенства Юнга (2.6), лемму 2.1 и оценки (4.5) и (4.6), $\forall u, v \in L_{2}(\mathbb{R})$ имеем

$$
\begin{gathered}
\|\Phi u-\Phi v\|_{2} \leqslant\left(m^{-1}+\|h\|_{1}\right) \cdot\|u-v\|_{2}, \\
\operatorname{Re}(\Phi u-\Phi v, u-v) \geqslant \frac{m}{M^{2}} \cdot\|u-v\|_{2}^{2} .
\end{gathered}
$$

Далее, заменяя уравнение 4.8 на эквивалентное уравнение

$$
v=\Psi v, \quad \text { где } \quad \Psi v=v-\mu \cdot(\Phi v-f), \quad \mu>0,
$$


как и при доказательстве теоремы 4.1, используя оценки 4.9) и 4.10, получим

$$
\begin{aligned}
\|\Psi u-\Psi v\|_{2}^{2} & =(u-v-\mu \cdot(\Phi u-\Phi v), u-v-\mu \cdot(\Phi u-\Phi v)) \\
& =\|u-v\|_{2}^{2}-2 \mu \cdot \operatorname{Re}(\Phi u-\Phi v, u-v)+\mu^{2} \cdot\|\Phi u-\Phi v\|_{2}^{2} \\
& \leqslant\left(1-2 \mu \cdot \frac{m}{M^{2}}+\mu^{2} \cdot\left(m^{-1}+\|h\|_{1}\right)^{2}\right) \cdot\|u-v\|_{2}^{2} .
\end{aligned}
$$

Из условий 4.1) и 4.2) вытекает, что $m \leqslant M$. Так как $-1 / m \leqslant-m / M^{2}$, то

$$
0 \leqslant 1-2 \mu \cdot \frac{1}{m}+\mu^{2} \cdot \frac{1}{m^{2}} \leqslant 1-2 \mu \cdot \frac{m}{M^{2}}+\mu^{2} \cdot\left(m^{-1}+\|h\|_{1}\right)^{2}<1,
$$

если

$$
\mu^{2} \cdot\left(m^{-1}+\|h\|_{1}\right)^{2}<2 \mu \cdot \frac{m}{M^{2}}, \quad \text { т.е. } \quad \mu<2 \frac{m}{M^{2}} \cdot \frac{1}{\left(m^{-1}+\|h\|_{1}\right)^{2}} .
$$

Поэтому, выбрав $\mu=\mu_{2}$, получим

$$
1-2 \mu \cdot \frac{m}{M^{2}}+\mu^{2} \cdot\left(m^{-1}+\|h\|_{1}\right)^{2}=1-m \cdot \mu_{2} / M^{2} .
$$

В результате, при указанном $\mu$ имеем:

$$
\|\Psi u-\Psi v\|_{2} \leqslant \alpha_{2} \cdot\|u-v\|_{2},
$$

где $\alpha_{2}=\sqrt{1-m \mu_{2} / M^{2}} \in(0,1)$.

Следовательно, на основании принципа сжимающих отображений, уравнение $v=\Psi v$, а значит, и уравнение (4.8), имеет единственное решение $v^{*}(x) \in L_{2}(\mathbb{R})$, причем последовательность

$$
v_{n}=\Psi v_{n-1}=v_{n-1}-\mu_{2} \cdot\left(H v_{n-1}+F^{-1} v_{n-1}-f\right),
$$

т.е. последовательность (4.3), сходится к $v^{*}(x)$ и

$$
\left\|v_{n}-v^{*}\right\|_{2} \leqslant \frac{\alpha_{2}^{n}}{1-\alpha_{2}} \cdot\left\|\Psi v_{0}-v_{0}\right\|_{2}=\mu_{2} \cdot \frac{\alpha_{2}^{n}}{1-\alpha_{2}} \cdot\left\|H v_{0}+F^{-1} v_{0}-f\right\|_{2} .
$$

Наконец, замечая, что $v^{*}=F u^{*}$, и используя неравенства (4.5), (4.6), для решения $u^{*}=F^{-1} v^{*} \in L_{2}(\mathbb{R})$ уравнения 3.2 получаем

$$
\begin{aligned}
\left\|u_{n}-u^{*}\right\|_{2} & =\left\|F^{-1} v_{n}-F^{-1} v^{*}\right\|_{2} \leqslant \frac{1}{m} \cdot\left\|v_{n}-v^{*}\right\|_{2} \\
& \leqslant \frac{\mu_{2}}{m} \cdot \frac{\alpha_{2}^{n}}{1-\alpha_{2}} \cdot\left\|H v_{0}+F^{-1} v_{0}-f\right\|_{2},
\end{aligned}
$$

т.е. справедливо неравенство 4.4.

Теорема 4.3. Пусть ядро $h \in L_{1}(\mathbb{R})$ и удовлетворяет условию (2.5). Если нелинейность $F(x, z)$ удовлетворяет условиям 4.1) и 4.2), то уравнение (3.3) имеет единственное решение $u^{*} \in L_{2}(\mathbb{R})$ при любом $f \in L_{2}(\mathbb{R})$. Это решение можно найти методом последовательных приближений по формуле:

$$
u_{n}=u_{n-1}+\mu_{2} \cdot\left(F^{-1}\left(f-u_{n-1}\right)-H u_{n-1}\right), \quad n \in \mathbb{N},
$$

где $\mu_{2}=m /\left[M \cdot\left(m^{-1}+\|h\|_{1}\right)\right]^{2}, F^{-1}$ есть оператор, обратный $\kappa F$. При этом справедлива оценка скорости сходимости последовательных приближений:

$$
\left\|u_{n}-u^{*}\right\|_{2} \leqslant \mu_{2} \cdot \frac{\alpha_{2}^{n}}{1-\alpha_{2}} \cdot\left\|F^{-1}\left(f-u_{0}\right)-H u_{0}\right\|_{2}
$$

где $\alpha_{2}=\sqrt{1-m \cdot \mu_{2} / M^{2}}, u_{0} \in L_{2}(\mathbb{R})-$ произвольная функиия. 
Доказательство. Запишем уравнение (3.3) в операторном виде

$$
u+F H u=f .
$$

В силу теоремы 3.4 оно имеет единственное решение $u^{*} \in L_{2}(\mathbb{R})$. Осталось доказать, что последовательность 4.12 сходится к $u^{*}$ и справедлива оценка (4.13). Для этого обозначим $f-u=v$. Тогда уравнение 4.14 примет вид $F H(f-v)=v$. Применив к обеим частям последнего уравнения оператор $F^{-1}$, существование которого установлено в доказательстве теоремы 4.2 , приходим к уравнению

$$
\Phi v=H f, \quad \text { где } \quad \Phi v=F^{-1} v+H v .
$$

т.е. к уравнению вида (4.8).

Далее, заменив уравнение 4.15 на эквивалентное

$$
v=B v, \quad \text { где } \quad B v=v-\mu \cdot(\Phi v-H f), \quad \mu>0,
$$

точно так же, как и при доказательстве теоремы 4.2, выбрав $\mu=\mu_{2}$, получим

$$
\|B u-B v\|_{2} \leqslant \alpha_{2} \cdot\|u-v\|_{2}
$$

Следовательно, на основании принципа сжимающих отображений, уравнение $v=B v$, а значит, и уравнение (4.5), имеет единственное решение $v^{*}=f-u^{*} \in L_{2}(\mathbb{R})$, причем последовательность

$$
v_{n}=v_{n-1}-\mu_{2} \cdot\left(\Phi v_{n-1}-H f\right)=v_{n-1}-\mu_{2} \cdot\left(F^{-1} v_{n-1}+H v_{n-1}-H f\right)
$$

сходится к $v^{*}$ и

$$
\left\|v_{n}-v^{*}\right\|_{2} \leqslant \mu_{2} \cdot \frac{\alpha_{2}^{n}}{1-\alpha_{2}} \cdot\left\|F^{-1} v_{0}+H v_{0}-H f\right\|_{2}
$$

Но тогда $u^{*}=f-v^{*} \in L_{2}(\mathbb{R})$ является (единственным) решением уравнения (4.14) и, в силу связи $v_{n}=f-u_{n}$, из 4.16 и 4.17 получаем

$$
\begin{aligned}
& f-u_{n}=f-u_{n-1}-\mu_{2} \cdot\left(F^{-1}\left(f-u_{n-1}\right)-H u_{n-1}\right), \\
& \left\|u_{n}-u^{*}\right\|_{2} \leqslant \mu_{2} \cdot \frac{\alpha_{2}^{n}}{1-\alpha_{2}} \cdot\left\|F^{-1}\left(f-u_{0}\right)-H u_{0}\right\|_{2},
\end{aligned}
$$

т.е. справедливы утверждения 4.12 и 4.13.

В заключение отметим, что аналогичные результаты можно получить (см. введение) в случае вещественных пространств $L_{p}(-\pi, \pi)$ без ограничений на $p \in(1, \infty)$, в отличие от теорем 3.2 3.4, а также для соответствующих нелинейных дискретных уравнений типа свертки как в вещественных, так и комплексных пространствах числовых последовательностей $l_{p}$ (см., соответственно, [21] и [22]). При этом важную роль играют условия положительности операторов свертки, приведенные в [16].

\section{СПИСОК ЛИТЕРАТУРЫ}

1. С.Н. Асхабов. Нелинейные уравнения типа свертки. М.: Физматлит. 2009.

2. H. Brunner. Volterra integral equations: an introduction to the theory and applications. Cambridge: Cambr. Univ. Press. 2017.

3. V.E. Beneš. A nonlinear integral equation from the theory of servo-mechanisms // Bell. System. Techn. J. 40:5, 1309-1321 (1961).

4. V.E. Beneš. A nonlinear integral equation in the Marcinkiewicz space $M_{2} / /$ J. Math. Phys. 44:1, 24-35 (1965).

5. O. Diekman. Thresholds and travelling waves for the geographical spread of infection // J. Math. Biol. 6:2, 109-130 (1978).

6. O. Diekman, H.G. Kaper. On the bounded solutions of nonlinear convolutions equation // Nonlinear Anal.: Theory, Meth. and Appl. 2:6, 721-737 (1978). 
7. Ф.Д. Гахов, Ю.И. Черский. Уравнения типа свертки. М.: Наука. 1978.

8. В. Хатсон, Дж. Пим. Приложения функционального анализа и теории операторов. М.: Мир. 1983.

9. М.А. Красносельский. Положителъные решения операторных уравнений. М.: Физматлит. 1962.

10. М.М. Вайнберг. Вариачионный метод и метод монотонных операторов в теории нелинейных уравнений. М.: Наука. 1972.

11. Х. Гаевский, К. Грегер, К. Захариас. Нелинейные операторные уравнения и операторные дифференциальные уравнения. М.: Мир. 1978.

12. С.Н. Асхабов. Периодические решения уравнений типа свертки с монотонной нелинейностъю // Уфимский матем. журнал. 8:1, 22-37 (2016).

13. Р. Эдвардс. Ряды Фуръе в современном изложении. Том 1. М.: Мир, 1985.

14. А.М. Нахушев. Дробное исчисление и его применение М.: Физматлит. 2003.

15. D. Porter, D. Stirling. Integral equations. A practical treatment, from spectral theory to applications. Cambridge: Cambr. Univ. Press. 1990.

16. S.N. Askhabov. Positivity Conditions for Operators with Difference Kernels in Reflexive Spaces // Journal of Math. Sciences. 250:5, 717-727 (2020).

17. А.Н. Колмогоров, С.В. Фомин. Элементы теории функций и функционального анализа. М.: Физматлит. 2004. 572 с.

18. Ю.А. Дубинский. Нелинейные эллиптические и параболические уравнения // Современные проблемы математики. Итоги науки и техники ВИНИТИ АН СССР. 9, 5-130 (1976).

19. H. Brezis, F.E. Browder. Some new results about Hammerstein equations // Bull. Amer. Math. Soc. 80:3, 567-572. (1974).

20. H. Brezis, F.E. Browder. Nonlinear integral equations and systems of Hammerstein type // Advances in Math. 18, 567-572 (1975).

21. С.Н. Асхабов, Н.К. Карапетянц. Дискретные уравнения типа свертки с монотонной нелинейностъю // Дифференц. уравнения. 25:10, 1777-1784 (1989).

22. S.N. Askhabov, N.K. Karapetian. Convolution Type Discrete Equations with Monotonous Nonlinearity in Complex Spaces // J. Integral Equat. Math. Phys. 1:1, 44-66 (1992).

Султан Нажмудинович Асхабов,

Чеченский государственный университет,

ул. Шерипова, 32,

364024, г. Грозный, Россия

Чеченский государственный педагогический университет, пр. Исаева, 62,

364068, г. Грозный, Россия

E-mail: askhabov@yandex.ru 